\title{
Sub-chronic toxicity evaluation of Dryopteris filix-mas (L.) schott, leaf extract in albino rats
}

\author{
Earnest Oghenesuvwe Erhirhie ${ }^{1, *}$, Emmanuel Emeka Ilodigwe ${ }^{1}$ \\ ${ }^{1}$ Department of Pharmacology and Toxicology, Faculty of Pharmaceutical Sciences, Nnamdi Azikiwe University, Awka, \\ Anambra State, Nigeria
}

\begin{abstract}
This study evaluated the acute and sub-chronic toxicities of ethanol leaf extract of Dryopteris filix-mas. Acute toxicity and phytochemical tests on ethanol leaf extract were determined. In sub-chronic toxicity test, animals were treated with $62.5,125,250$ and $500 \mathrm{mg} / \mathrm{kg}$ of extract every day for 90 days. Blood samples were collected via retro-orbital puncture for baseline studies and at 31,61 and $91^{\text {st }}$ days for determination of hematological, kidney and liver function parameters. Liver and kidneys were harvested for histopathology analyses on $91^{\text {st }}$ day. Also, a 28 day recovery study was carried out to determine reversibility in toxicological effects. Phytochemical screening revealed the presence of tannins, phenols, flavonoids, saponins, steroids, alkaloids, terpenoids, reducing sugar and cardiac glycosides. Acute toxicity test did not show toxicity or death at $5000 \mathrm{mg} / \mathrm{kg}$. There was significant $(\mathrm{p}<0.005)$ reduction in white blood cell and lymphocyte counts, significant $(\mathrm{p}<0.05)$ increase in some liver and kidney biomarkers as well as alterations in liver and kidney histo-architecture on $91^{\text {st }}$ days in animals that were treated with 250 and $500 \mathrm{mg} / \mathrm{kg}$ extract. However, toxicities observed on $91^{\text {st }}$ day were reversible in recovery studies. The leaf extract of Dryopteris filix-mas may be hepatotoxic and nephrotoxic when used for long periods.
\end{abstract}

Keywords: Dryopteris filix mas Leaf Extract. Sub-chronic toxicity. Acute toxicity. Nephrotoxicity. Hepatotoxicity. Dryopteris/toxicity. Toxicity tests/utilization. Ethanol/ toxicity.

\section{INTRODUCTION}

Medicinal plants are the richest alternative bioresources to synthetic drugs (Obi et al., 2012). Presently, there is a revitalization of interest in the use of medicinal plants by over $80 \%$ of the world population (Ogbonnia et al., 2011). In African countries, a large proportion of the population depends solely on herbal medicines for their primary health care needs. This is because medicinal plants are accessible, acceptable and affordable among the populace when compared to synthetic drugs (Obi et al., 2012).

Dryopteris filix-mas, Dryoperidaceae is a deciduous evergreen perennial herb growing up to $60-150 \mathrm{~cm}$ tall, with rhizomes. It is an effective ground cover plant that is native to Europe, Asia, and North America. It has a large light-green triangular frond (Sekendar et al., 2012). It is commonly found in streams, shady places and

\footnotetext{
*Correspondence: Earnest Oghenesuvwe Erhirhie. Department of Pharmacology and Toxicology, Faculty of Pharmaceutical Sciences, Nnamdi Azikiwe University, Agulu, Anambra State, Nigeria. Tel.: +234-7060434974. E-mail: erhirhieochuko@yahoomail.com (iD)
}

wetland areas. It is commonly referred to as male fern, Aspidium, water loving fern, worm fern and shield fern (Uwumarongie, Enike, Bafor, 2016). Its vernacular names include; fougère mâle (local French), sarkhas; shurud (Arabic), Eraketa (Urhobo), and Akpaka or Akolor (Igbo). The edible parts of Dryopteris filix-mas include; the leaves and root. The rhizomes can be eaten raw or cooked as part of a regimen for losing weight. In traditional medicine, the leaves and rhizome are used in the treatment of rheumatoid arthritis, inflammation, malaria, worm infestation, internal haemorrhage, uterine bleeding, fever, mumps, carbuncles and sores (Sekendar et al., 2012). The leaves, roots and rhizomes are used to rinse hair and to treat dandruffs (Sekendar et al., 2012; Soare et al., 2012). Literature had reported its anti-diarrheal activity (Uwumarongie, Enike, Bafor, 2016), antioxidant and cytotoxic activities (Sekendar et al., 2012), anti-helmintic activity (Urban et al., 2014) and anti-microbial activity (Soare et al., 2012).

Following the ethnomedicinal values of Dryopteris filix-mas in Nigeria, there are no scientific literatures to validate its safety profile due to its repeated use overtime in the treatment and management of various diseases. The 
main purpose of this study is to evaluate the sub-chronic toxicities of Dryopteris filix-mas leaf extract on some toxicological parameters.

\section{MATERIAL AND METHODS}

\section{Material}

Equipments and apparatuses used in this study include; visible Spectrophotometer $(721 \mathrm{G}$, Zhejiang Top Cloud-Agri Technology Co., Ltd., China), Abacus Junior hematology Analyzer (Diatron Abacus 380, Hungary), table Centrifuge, thermostatic water bath (Equitron Mumbai India), analytical weighing balance (Ohaus Corp. Pine Brook, NJ USA), animal cages and micro pipettes.

\section{Chemicals and reagents}

Chemicals and reagents used in this study include sodium hydroxide pellets (Avondale Laboratories supplies and Services, England), Ethanol (JHD, Guangdong Guanghua Schi-Tech), Formaldehyde (May and Baker Ltd, Dagenham England). Aspartate aminotransferase (AST), Alanine aminotransferase (ALT), Triglyceride, Total cholesterol, High density lipoprotein cholesterol (HDL-c) reagent kits were procured from Randox Laboratories Limited, Country Atrium, United Kingdom while Sodium, Chloride, Potassium, Urea, Creatinine, Total protein, Albumin and Alkaline phosphatase (ALP) kits were procured from Teco diagnostics, California U.S.A.

\section{Experimental animals}

Albino rats of either sex were procured from Department of Veterinary Medicine, University of Nigeria Nnsukka. They were kept in the animal house, Department of Pharmacology and Toxicology, Faculty of Pharmaceutical Sciences, Nnamdi Azikiwe University, Agulu and were given access to water and pelletized vital grower feed ad libitum under 12:12 hours light and dark cycle. Animals were handled in conformity with the National Institute of Health Guidelines for the care and use of laboratory animals for research purpose (Pub No. 85-23, revised 1985).

\section{Plant collection and authentication}

Fresh leaves of Dryopteris filix-mas were collected between 6:30 and 8:00 am in the month of March, 2016 from a swampy area beside the Horticulture botanical garden, Amawbia, Awka South L.G.A, Anambra State, Nigeria. Plant specimen was validated by Dr. Akinnibosun H.A, a plant taxonomist of the Department of Plant Biology and Biotechnology, Faculty of Life Sciences, University of Benin, Benin City, Nigeria. It was deposited in the herbarium of the Department and Voucher number " $\mathrm{UBH}_{\mathrm{d}} 285 \mathrm{~A}$ " was assigned to it.

\section{Preparation of plant extract}

The extract was prepared using maceration method described by Azubike et al. (2015). Fresh leaves of Dryopteris filix-mas were washed with tap water and airdried at room temperature for one week. Crisply dried leaves were pulverized using mechanical grinder and a total of $4.7 \mathrm{~kg}$ was extract by cold-macerated using $80 \%$ aqueous ethanol for a period of 48 hour, with occasional agitation. Filtrate was recovered with the aid of a muslin clothe. Final filtrate recovered was concentrated using water bath at $40{ }^{\circ} \mathrm{C}$ until a greenish paste was formed.

\section{Phytochemical screening}

The extract was screened for the presence of proteins, reducing sugars, tannins, flavonoids, saponins, cardiac glycosides, steroids, terpenoids, anthraquinolones and alkaloids using methods of Sofowora (1993), Trease, Evans (1989) and Harborne (1973).

\section{Acute toxicity test}

Acute toxicity test on the extract was carried out in rats using the method of Miller and Tainter as described by Randhawa (2009). Sixty 60 albino rats $(129.86 \pm 0.98 \mathrm{~g}$ body weight) of either sex were grouped into 6 groups of 10 animals each as follows; control, 100, 1000, 2000, 3000 and $5000 \mathrm{mg} / \mathrm{kg}$ of extract. The control group was given $10 \mathrm{~mL} / \mathrm{kg}$ of distilled water. Observation was made during the first 4 hours and after 24 hours for signs of toxicity or death. Also, 2 weeks observation was given to the animals for signs of delayed toxicity and death.

\section{Sub-chronic toxicological studies}

The protocol described by Ilodigwe, Akah, Nworu (2010) was used in this study with some modifications. A total of sixty (60) albino rats of either sex $(84.00 \pm 2.89 \mathrm{~g}$ body weight) were randomized into five groups of twelve animals each as follows: Control group $(10 \mathrm{~mL} / \mathrm{kg}$, distilled water), and test groups $(62.5,125,250$ and 500 
$\mathrm{mg} / \mathrm{kg}$ of the extract). The extract was reconstituted in distilled water to form various concentrations, $50 \mathrm{mg} / \mathrm{mL}$, $25 \mathrm{mg} / \mathrm{mL}, 12.5 \mathrm{mg} / \mathrm{mL}$ and $6.25 \mathrm{mg} / \mathrm{mL}$ for 500,250 , 125 and $62.5 \mathrm{mg} / \mathrm{kg}$ respectively. Blood samples were collected via the retro-orbital plexus for determination of baseline hematological parameters using Abacus Junior Hematology Analyzer (Diatron Abacus 380). Serum recovered from blood samples collected into plain tube and centrifuged at $3500 \mathrm{rpm}$ for 10 minutes was diluted 5-fold with normal saline and used for the assay of biochemical parameters including alkaline phosphatase (ALP), alanine aminotransferases (ALT), aspartate transaminases (AST), total protein, albumin, sodium, potassium, chloride, calcium, urea, creatinine, total cholesterol, triglyceride and high density lipoprotein cholesterol using manufacturers' (Randox and Teco) kits leaflets procedures with little modifications. Normal saline was added to reagent blank and the resulting absorbance of sample was multiplied by five. After baseline assessment, animals were dosed daily with the extract for a period of 90-days. On $31^{\text {st }}$, $61^{\text {st }}$ and $91^{\text {st }}$ days, blood samples collected from retroorbital plexus into EDTA tubes and plain tubes were used for determination of hematological and biochemical parameters respectively using similar protocols described for baseline studies. Body weight gain and organ weights were recorded. Liver and kidney were fixed in $10 \%$ formal saline for histopathological analyses using the method described by Bancroft, Gamble (2002). Photomicrographs were captured and interpreted by a histopathologist at $\times 400$ magnifications using Microscope attached to a digital camera. Animals' stomachs were removed and cut open along the lesser curvature and washed with tap water and observed for presence or absence of lesions using the method described by Moke, Ilodigwe, Erhirhie (2015).

\section{Recovery studies}

At the end of 90-days, animals were placed on feed and water ad-libitum without extract administration for 28 -days. At the end of 28 days (day $29^{\text {th }}$ ), blood samples collected from retro-orbital plexus of animals were used for the determination of hematological and biochemical parameters. Body weights and organ weights were recorded. Liver and kidney were fixed in $10 \%$ formal saline for histopathological analyses.

\section{Method of data analyses}

Results were presented as mean \pm Standard error of mean (SEM) of sample replicates $(n=5)$. Raw data were analyzed using one way analyses of variance (ANOVA), followed by post hoc Turkey's test using Statistical Package for Social Science (SPSS, version 20). $p<0.05$ was established to be statistically significant.

\section{RESULTS AND DISCUSSION}

This study evaluated the sub-chronic toxicity profile of Dryopteris filix-mas in albino rats. From $\mathrm{LD}_{50}$ study, there were no signs of toxicity or death recorded at various doses $(100,1000,2000,3000$ and $5000 \mathrm{mg} / \mathrm{kg})$ of the extract after 24 hours and subsequently for 14-days when compared to control group. This suggests that the extract is not toxic on short term exposure in this experimental condition. $\mathrm{LD}_{50}$ value above $5000 \mathrm{mg} / \mathrm{kg}$ is classified as non-toxic (Muhammad et al., 2015). Phytochemical screening revealed the presence of tannins, flavonoids, saponins, steroids, alkaloids, terpenoids and reducing sugars in the leaf extract of Dryopteris filix-mas (Table I). These secondary metabolites have been shown to exert various toxicological effects apart from their benefits (Aksel, 2010; Yadav, Agarwala, 2011). In support of this finding, Uwumarongie and co-workers in the study of the phytochemical constituents of Dryopteris filix mas leaf also reported the presence of glycosides, tannins, flavonoids, steroids and other nutrients (Uwumarongie, Enike, Bafor, 2016).

TABLE I - Qualitative phytochemistry results

\begin{tabular}{lc}
\hline Phytochemicals & Results \\
\hline Tannins & ++ \\
Flavonoids & +++ \\
Saponins & ++ \\
Steroids & ++ \\
Alkaloids & ++ \\
Terpenoids & ++ \\
Anthraquinolones & - \\
Cardiac glycosides & + \\
Reducing sugars & + \\
\hline “-":absent, "+" :trace “++": moderate and “+++": abundant.
\end{tabular}

Hematological parameters assessment is vital among various toxicity biomarkers to know whether test substances affect the hematopoitic system (Bashir et al., 2015). From Table II, there was significant $(* p<0.05)$ increase in PCV, RBC and hemoglobin on $31^{\text {st }}$ day following the administration of 250 and $500 \mathrm{mg} / \mathrm{kg}$ of Dryopteris filix-mas leaf extract to albino rats. This suggests that Dryopteris filix-mas could promote red 
blood cell production at 250 and $500 \mathrm{mg} / \mathrm{kg}$ doses after 30 days of its exposure to animals. Plant secondary metabolites such as flavonoids and terpenoids have been reported to promote erythropoisis (Osano et al., 2016) and presence of these metabolites in the extract (Table I) may account for increase in PCV, RBC and hemoglobin on day $31^{\text {st }}$. Non-significant changes in PCV, hemoglobin and RBC levels on 61 and $91^{\text {st }}$ days suggest that the extract does not promote red blood cell production at 250 and $500 \mathrm{mg} / \mathrm{kg}$ after longer duration of exposure. Long term intake of medicinal plants rich in saponins had been reported to cause hemolysis of red blood cells (Ekpenyong, Akpan, Udoh, 2012). Study by Kumar, Karthik, Rao (2011) also revealed that presence of saponins in extracts of some Indian medicinal plants resulted in hemolytic effects due to alteration in the erythrocyte membrane. Thus, presence of saponins in the extract may account for the reversibility in high PCV, RBC and hemoglobin levels of $31^{\text {st }}$ day on 61 and $91^{\text {st }}$ days.

From Table III, there was significant $(* p<0.05)$ decrease in WBC and lymphocyte counts and significant $(* p<0.05)$ increase in granulocyte and medium size cells

TABLE II - Effect of sub-chronic administration of Dryopteris filix-mas ethanol leaf extract on packed cell volume (PCV), red blood cell (RBC), hemoglobin and platelet (PLAT) levels of albino rats

\begin{tabular}{|c|c|c|c|c|c|}
\hline & Treatment & PCV (\%) & RBC $\left(10^{6} / \mu \mathrm{L}\right)$ & Hemoglobin (g/dL) & $\operatorname{PLAT}\left(10^{3} / \mu \mathrm{L}\right)$ \\
\hline & Control & $45.66 \pm 1.00$ & $7.49 \pm 1.54$ & $14.66 \pm 0.29$ & $864.40 \pm 41.48$ \\
\hline & $61.25 \mathrm{mg} / \mathrm{kg}$ & $43.03 \pm 1.61$ & $7.24 \pm 0.22$ & $15.14 \pm 0.51$ & $847.80 \pm 37.47$ \\
\hline \multirow[t]{5}{*}{ Baseline } & $125 \mathrm{mg} / \mathrm{kg}$ & $44.78 \pm 0.74$ & $7.18 \pm 0.16$ & $15.46 \pm 0.49$ & $832.20 \pm 39.55$ \\
\hline & $250 \mathrm{mg} / \mathrm{kg}$ & $44.46 \pm 1.50$ & $7.51 \pm 0.16$ & $14.58 \pm 0.41$ & $795.60 \pm 46.06$ \\
\hline & $500 \mathrm{mg} / \mathrm{kg}$ & $42.56 \pm 0.93$ & $7.35 \pm 0.20$ & $15.46 \pm 0.27$ & $847.40 \pm 32.45$ \\
\hline & Control & $41.03 \pm 1.33$ & $6.24 \pm 0.30$ & $14.74 \pm 0.57$ & $677.80 \pm 54.22$ \\
\hline & $61.25 \mathrm{mg} / \mathrm{kg}$ & $39.97 \pm 1.68$ & $6.58 \pm 0.25$ & $14.05 \pm 0.70$ & $677.80 \pm 48.59$ \\
\hline \multirow[t]{5}{*}{ Day $31^{\text {st }}$} & $125 \mathrm{mg} / \mathrm{kg}$ & $39.77 \pm 0.96$ & $6.34 \pm 0.28$ & $13.86 \pm 0.28$ & $618.60 \pm 37.94$ \\
\hline & $250 \mathrm{mg} / \mathrm{kg}$ & $54.56 \pm 3.61^{*}$ & $8.39 \pm 0.12^{*}$ & $19.55 \pm 1.31 *$ & $664.00 \pm 43.81$ \\
\hline & $500 \mathrm{mg} / \mathrm{kg}$ & $54.34 \pm 4.81^{*}$ & $8.55 \pm 0.19^{*}$ & $19.34 \pm 1.79 *$ & $786.60 \pm 86.02$ \\
\hline & Control & $41.18 \pm 0.67$ & $6.63 \pm 0.14$ & $14.20 \pm 0.21$ & $675.20 \pm 77.68$ \\
\hline & $61.25 \mathrm{mg} / \mathrm{kg}$ & $40.44 \pm 0.65$ & $6.63 \pm 0.15$ & $13.88 \pm 0.16$ & $667.40 \pm 66.13$ \\
\hline \multirow[t]{5}{*}{ Day $61^{\text {st }}$} & $125 \mathrm{mg} / \mathrm{kg}$ & $40.28 \pm 1.50$ & $6.52 \pm 0.27$ & $14.02 \pm 0.54$ & $633.40 \pm 29.53$ \\
\hline & $250 \mathrm{mg} / \mathrm{kg}$ & $41.14 \pm 1.13$ & $6.70 \pm 0.15$ & $14.54 \pm 0.41$ & $561.00 \pm 25.80$ \\
\hline & $500 \mathrm{mg} / \mathrm{kg}$ & $40.77 \pm 0.56$ & $6.64 \pm 0.14$ & $13.86 \pm 0.16$ & $599.20 \pm 27.20$ \\
\hline & Control & $42.74 \pm 1.01$ & $7.00 \pm 0.22$ & $13.68 \pm 0.35$ & $760.80 \pm 42.92$ \\
\hline & $61.25 \mathrm{mg} / \mathrm{kg}$ & $42.47 \pm 0.45$ & $6.77 \pm 0.37$ & $13.99 \pm 0.12$ & $681.20 \pm 23.22$ \\
\hline \multirow[t]{5}{*}{ Day $91^{\text {st }}$} & $125 \mathrm{mg} / \mathrm{kg}$ & $43.71 \pm 0.38$ & $6.89 \pm 0.07$ & $14.06 \pm 0.17$ & $821.00 \pm 54.83$ \\
\hline & $250 \mathrm{mg} / \mathrm{kg}$ & $42.19 \pm 1.06$ & $6.96 \pm 0.16$ & $13.12 \pm 0.35$ & $780.40 \pm 30.97$ \\
\hline & $500 \mathrm{mg} / \mathrm{kg}$ & $44.26 \pm 1.83$ & $7.15 \pm 0.32$ & $13.62 \pm 0.60$ & $837.40 \pm 37.43$ \\
\hline & Control & $43.48 \pm 1.18$ & $6.94 \pm 0.29$ & $13.12 \pm 0.39$ & $797.80 \pm 68.75$ \\
\hline & $61.25 \mathrm{mg} / \mathrm{kg}$ & $43.28 \pm 0.94$ & $6.95 \pm 0.21$ & $13.38 \pm 0.36$ & $793.20 \pm 47.88$ \\
\hline \multirow[t]{3}{*}{ Recovery } & $125 \mathrm{mg} / \mathrm{kg}$ & $44.65 \pm 1.36$ & $7.11 \pm 0.29$ & $13.66 \pm 0.46$ & $707.80 \pm 82.30$ \\
\hline & $250 \mathrm{mg} / \mathrm{kg}$ & $42.59 \pm 0.82$ & $6.72 \pm 0.23$ & $12.92 \pm 0.34$ & $734.60 \pm 70.48$ \\
\hline & $500 \mathrm{mg} / \mathrm{kg}$ & $44.89 \pm 0.89$ & $7.04 \pm 0.11$ & $13.73 \pm 0.19$ & $835.40 \pm 33.18$ \\
\hline
\end{tabular}

Values are presented as mean \pm Standard error of mean $(n=5) .{ }^{*} p<0.05$ : Statistically significantly different from control group. 
count on $91^{\text {st }}$ day following the administration of 250 and $500 \mathrm{mg} / \mathrm{kg}$ of Dryopteris filix-mas leaf extract to albino rats. This suggests that bioaccumulation of the extract due to its 90 days exposure to animals could disrupt bone marrow function resulting to insufficient production of leukocytes. Debelo et al. (2016) stated that reduction in WBC level is associated with immune suppression. Studies by Unakalamba, Ozougwu, Ejere (2013) revealed that saponins in medicinal plants could cause decrease production in WBC. Usually, reduction in total leukocyte and lymphocyte count correlates with increase in MID and granulocyte count (Yadav et al., 2010). Non significant change in these parameters following 28 days recovery studies suggests that leukocyte suppression effect of the extract was reversible.

From Table IV, there was no significant difference $(\mathrm{p}>0.05)$ in PCT, MPV, MCV, MCH, and MCHC levels on 31,61 and $91^{\text {st }}$ days in animals treated with various doses $(62.5,125,250$ and $500 \mathrm{mg} / \mathrm{kg})$ of Dryopteris filix-mas leaf extract. This indicates that components of these blood parameters may not be deleteriously affected by the extract.

TABLE III - Effect of sub-chronic administration of Dryopteris filix-mas ethanol leaf extract on white blood cell (WBC), lymphocyte (Lymp), granulocyte (Gran), and medium size cell counts (MID) of albino rats

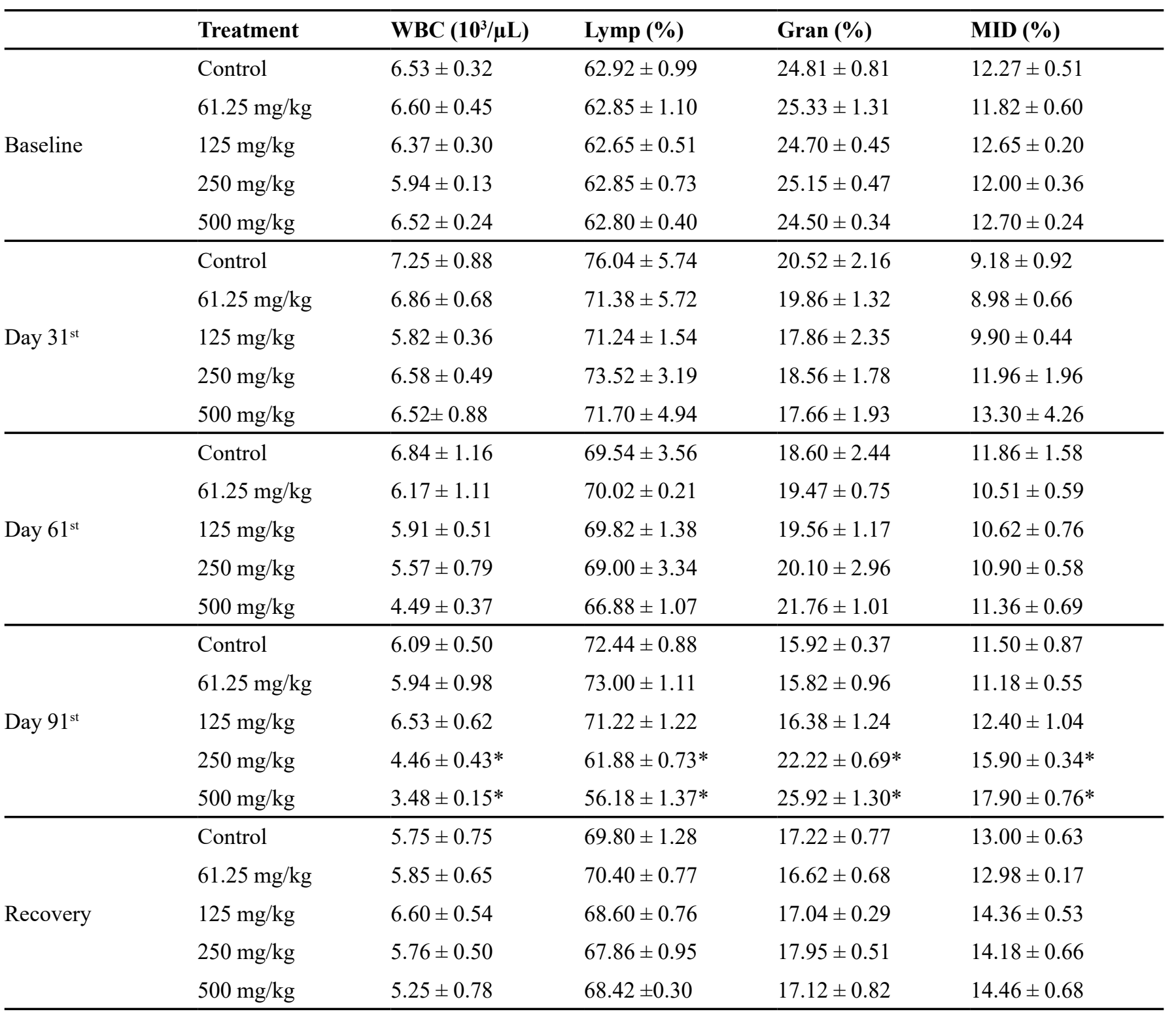

Values are presented as mean \pm Standard error of mean $(n=5) .{ }^{*}<0.05$ : Statistically significantly different from control group. 
Increase in cholesterol and triglyceride levels could be associated with cardiovascular and coronary heart disease (Kayode et al., 2016). From this study, significant reduction in total cholesterol and triglyceride levels at $250 \mathrm{mg} / \mathrm{kg}$ dose on day 61 (Table V) suggests that 250 $\mathrm{mg} / \mathrm{kg}$ dose could promote lipid lowering. Presence of saponins and flavonoids in medicinal plants have been reported to play significant roles in cholesterol and lipid lowering (Yadav, Agarwala, 2011; Builders, Isichie, Aguiyi, 2012).

Liver enzymes (AST, ALT, ALP), total protein and albumin concentrations are considered when assessing liver function. Therefore, alteration in the level of these biomarkers could be an indication of liver injury. When the liver is exposed to toxicants, damage to its membrane may occur, thereby causing leakages and elevation in the level of these enzymes in the blood circulation. On the other hand, whenever the liver is protected, its membrane remains intact thereby causing non-alteration or reduction in its enzyme levels (Otunola, Afolayan, 2017).

From Table VI, significant increase in liver enzymes (ALT, AST and ALP) in 250 and $500 \mathrm{mg} / \mathrm{kg}$ groups on $91^{\text {st }}$

TABLE IV - Effect of sub-chronic administration of Dryopteris filix-mas ethanol leaf extract on platelet percentage (PCT), mean platelet volume (MPV), mean corpuscular volume (MCV), mean corpuscular hemoglobin (MCH), and mean corpuscular hemoglobin concentration (MCHC) of albino rats

\begin{tabular}{|c|c|c|c|c|c|c|}
\hline & Treatment & PCT (\%) & MPV (fL) & MCV (fL) & MCH (pg) & $\operatorname{MCHC}(\mathrm{g} / \mathrm{dL})$ \\
\hline & Control & $0.97 \pm 0.04$ & $8.94 \pm 0.17$ & $65.00 \pm 0.45$ & $21.04 \pm 0.17$ & $32.14 \pm 0.52$ \\
\hline & $61.25 \mathrm{mg} / \mathrm{kg}$ & $0.88 \pm 0.04$ & $8.76 \pm 0.09$ & $64.80 \pm 0.97$ & $20.74 \pm 0.37$ & $32.72 \pm 0.67$ \\
\hline \multirow[t]{5}{*}{ Baseline } & $125 \mathrm{mg} / \mathrm{kg}$ & $0.83 \pm 0.02$ & $8.66 \pm 0.26$ & $64.40 \pm 0.87$ & $20.94 \pm 0.39$ & $32.62 \pm 0.63$ \\
\hline & $250 \mathrm{mg} / \mathrm{kg}$ & $0.81 \pm 0.06$ & $8.70 \pm 0.61$ & $63.60 \pm 0.68$ & $20.36 \pm 0.57$ & $32.74 \pm 0.45$ \\
\hline & $500 \mathrm{mg} / \mathrm{kg}$ & $0.95 \pm 0.05$ & $8.48 \pm 0.25$ & $65.00 \pm 0.63$ & $20.50 \pm 0.29$ & $33.54 \pm 0.32$ \\
\hline & Control & $0.52 \pm 0.04$ & $7.72 \pm 0.24$ & $63.20 \pm 1.02$ & $20.84 \pm 0.35$ & $33.12 \pm 0.28$ \\
\hline & $61.25 \mathrm{mg} / \mathrm{kg}$ & $0.53 \pm 0.05$ & $7.60 \pm 0.2$ & $63.4 \pm 1.28$ & $20.84 \pm 0.53$ & $32.56 \pm 0.87$ \\
\hline \multirow[t]{5}{*}{ Day $31^{\text {st }}$} & $125 \mathrm{mg} / \mathrm{kg}$ & $0.47 \pm 0.02$ & $7.56 \pm 0.14$ & $62.60 \pm 1.08$ & $20.12 \pm 0.35$ & $32.16 \pm 0.24$ \\
\hline & $250 \mathrm{mg} / \mathrm{kg}$ & $0.54 \pm 0.03$ & $8.16 \pm 0.17$ & $66.40 \pm 2.42$ & $21.54 \pm 0.52$ & $32.42 \pm 0.47$ \\
\hline & $500 \mathrm{mg} / \mathrm{kg}$ & $0.62 \pm 0.07$ & $7.98 \pm 0.21$ & $63.40 \pm 1.57$ & $20.94 \pm 0.69$ & $33.10 \pm 0.59$ \\
\hline & Control & $0.71 \pm 0.07$ & $7.74 \pm 0.24$ & $62.00 \pm 0.71$ & $21.46 \pm 0.22$ & $34.48 \pm 0.14$ \\
\hline & $61.25 \mathrm{mg} / \mathrm{kg}$ & $0.68 \pm 0.05$ & $7.64 \pm 0.30$ & $63.40 \pm 1.03$ & $21.38 \pm 0.42$ & $33.62 \pm 0.60$ \\
\hline \multirow[t]{5}{*}{ Day $61^{\text {st }}$} & $125 \mathrm{mg} / \mathrm{kg}$ & $0.65 \pm 0.02$ & $7.30 \pm 0.07$ & $61.80 \pm 1.02$ & $21.50 \pm 0.38$ & $34.80 \pm 0.24$ \\
\hline & $250 \mathrm{mg} / \mathrm{kg}$ & $0.63 \pm 0.03$ & $7.70 \pm 0.18$ & $61.40 \pm 0.68$ & $21.68 \pm 0.58$ & $35.30 \pm 0.70$ \\
\hline & $500 \mathrm{mg} / \mathrm{kg}$ & $0.65 \pm 0.02$ & $7.64 \pm 0.09$ & $61.60 \pm 1.72$ & $20.94 \pm 0.54$ & $34.02 \pm 0.14$ \\
\hline & Control & $0.60 \pm 0.04$ & $7.84 \pm 0.09$ & $61.20 \pm 1.74$ & $19.54 \pm 0.21$ & $32.02 \pm 0.70$ \\
\hline & $61.25 \mathrm{mg} / \mathrm{kg}$ & $0.60 \pm 0.05$ & $7.48 \pm 0.12$ & $60.8 \pm 1.31$ & $19.37 \pm 0.28$ & $32.01 \pm 0.83$ \\
\hline \multirow[t]{5}{*}{ Day $91^{\text {st }}$} & $125 \mathrm{mg} / \mathrm{kg}$ & $0.65 \pm 0.05$ & $7.94 \pm 0.14$ & $63.50 \pm 0.50$ & $20.42 \pm 0.19$ & $32.18 \pm 0.16$ \\
\hline & $250 \mathrm{mg} / \mathrm{kg}$ & $0.66 \pm 0.02$ & $8.42 \pm 0.29$ & $60.60 \pm 1.53$ & $18.90 \pm 0.39$ & $31.12 \pm 0.20$ \\
\hline & $500 \mathrm{mg} / \mathrm{kg}$ & $0.68 \pm 0.04$ & $8.16 \pm 0.17$ & $62.20 \pm 1.02$ & $19.08 \pm 0.42$ & $30.78 \pm 0.32$ \\
\hline & Control & $0.63 \pm 0.06$ & $7.84 \pm 0.08$ & $60.20 \pm 0.37$ & $21.40 \pm 0.25$ & $32.25 \pm 0.27$ \\
\hline & $61.25 \mathrm{mg} / \mathrm{kg}$ & $0.63 \pm 0.04$ & $7.84 \pm 0.23$ & $61.60 \pm 0.51$ & $20.20 \pm 0.20$ & $32.25 \pm 0.34$ \\
\hline \multirow[t]{3}{*}{ Recovery } & $125 \mathrm{mg} / \mathrm{kg}$ & $0.58 \pm 0.06$ & $7.72 \pm 0.14$ & $62.80 \pm 0.86$ & $20.88 \pm 0.35$ & $32.18 \pm 0.54$ \\
\hline & $250 \mathrm{mg} / \mathrm{kg}$ & $0.57 \pm 0.06$ & $7.82 \pm 0.17$ & $61.20 \pm 0.86$ & $20.32 \pm 0.50$ & $31.23 \pm 0.44$ \\
\hline & $500 \mathrm{mg} / \mathrm{kg}$ & $0.66 \pm 0.04$ & $7.90 \pm 0.31$ & $63.60 \pm 0.60$ & $22.30 \pm 1.03$ & $32.38 \pm 0.39$ \\
\hline
\end{tabular}

Values are presented as mean \pm Standard error of mean $(n=5) . p>0.05$ : Not statistically significantly different from control group. 
day suggests that the extract could cause hepatic injury when used for long duration. These biochemical changes were corroborated by the liver histopathology results (Figure 1) characterized by congestion of the hepatic triad leading to displacement of red blood cells from the intravascular space to the loose connective tissues in dose dependent manner. In recovery studies, significant increase in liver enzymes, as well as histopathological changes observed on $91^{\text {st }}$ day were not observed (Table VI and Figure 2), suggesting that the injuries attributed to long term exposure to the extract for 90 days was reversible.

Electrolytes (sodium, potassium, chloride and calcium), urea and creatinine are put into consideration when assessing kidney function. Increase in renal biomarkers as well as alteration in kidney cytoarchitecture is an indication of compromised renal functions (Ogbonnaya, Uadia, 2016).

Non-significant change in electrolytes and total protein (Table VII) suggests that the physiological function of these biomarkers may not be hampered following the intake of the extract for 90 days.

However, significant increase in kidney function parameters, urea and creatinine (Table VIII) on the $91^{\text {st }}$ day suggests that the extract could be associated with kidney injury due to its longer duration of exposure. These changes were corroborated by the kidney histopathology

TABLE V - Effect of sub-chronic administration of Dryopteris filix-mas ethanol leaf extract on Lipid profile of albino rats

\begin{tabular}{|c|c|c|c|c|c|}
\hline & Treatment & $\begin{array}{c}\text { Total cholesterol } \\
(\mathrm{mg} / \mathrm{dL})\end{array}$ & $\begin{array}{c}\text { Triglyceride } \\
\text { (mg/dL) }\end{array}$ & $\begin{array}{c}\text { HDL-cholesterol } \\
(\mathrm{mg} / \mathrm{dL})\end{array}$ & $\begin{array}{c}\text { LDL-cholesterol } \\
\text { (mg/dL) }\end{array}$ \\
\hline \multirow{5}{*}{ Baseline } & Control & $148.17 \pm 3.39$ & $114.34 \pm 2.51$ & $51.85 \pm 2.03$ & $73.46 \pm 3.05$ \\
\hline & $61.25 \mathrm{mg} / \mathrm{kg}$ & $149.27 \pm 1.89$ & $113.13 \pm 3.53$ & $52.92 \pm 3.06$ & $73.73 \pm 4.38$ \\
\hline & $125 \mathrm{mg} / \mathrm{kg}$ & $141.52 \pm 2.02$ & $113.49 \pm 2.55$ & $49.39 \pm 3.42$ & $69.43 \pm 3.42$ \\
\hline & $250 \mathrm{mg} / \mathrm{kg}$ & $146.00 \pm 2.36$ & $111.17 \pm 2.81$ & $50.68 \pm 4.42$ & $73.08 \pm 3.88$ \\
\hline & $500 \mathrm{mg} / \mathrm{kg}$ & $146.93 \pm 2.16$ & $108.78 \pm 2.79$ & $52.55 \pm 3.58$ & $72.62 \pm 4.71$ \\
\hline \multirow{5}{*}{ Day $31^{\text {st }}$} & Control & $142.02 \pm 5.37$ & $111.24 \pm 3.05$ & $57.18 \pm 1.23$ & $62.59 \pm 5.78$ \\
\hline & $61.25 \mathrm{mg} / \mathrm{kg}$ & $130.82 \pm 3.89$ & $114.16 \pm 2.79$ & $57.65 \pm 1.77$ & $50.33 \pm 5.23$ \\
\hline & $125 \mathrm{mg} / \mathrm{kg}$ & $141.03 \pm 8.28$ & $110.45 \pm 2.60$ & $59.91 \pm 1.23$ & $59.02 \pm 8.06$ \\
\hline & $250 \mathrm{mg} / \mathrm{kg}$ & $132.13 \pm 7.98$ & $112.57 \pm 4.83$ & $60.63 \pm 0.87$ & $48.99 \pm 7.04$ \\
\hline & $500 \mathrm{mg} / \mathrm{kg}$ & $135.43 \pm 3.95$ & $112.04 \pm 1.36$ & $60.99 \pm 1.15$ & $52.03 \pm 4.82$ \\
\hline \multirow{5}{*}{ Day $61^{\text {st }}$} & Control & $140.71 \pm 6.33$ & $117.47 \pm 4.52$ & $66.67 \pm 1.84$ & $50.55 \pm 7.63$ \\
\hline & $61.25 \mathrm{mg} / \mathrm{kg}$ & $115.47 \pm 6.88$ & $118.10 \pm 3.40$ & $71.59 \pm 2.43$ & $42.44 \pm 5.72$ \\
\hline & $125 \mathrm{mg} / \mathrm{kg}$ & $125.41 \pm 3.71$ & $95.84 \pm 7.75$ & $74.62 \pm 7.08$ & $31.63 \pm 9.10$ \\
\hline & $250 \mathrm{mg} / \mathrm{kg}$ & $115.47 \pm 6.88^{*}$ & $81.22 \pm 4.70 *$ & $68.56 \pm 3.14$ & $30.67 \pm 5.98$ \\
\hline & $500 \mathrm{mg} / \mathrm{kg}$ & $126.18 \pm 6.51$ & $98.87 \pm 5.57$ & $75.00 \pm 2.21$ & $31.40 \pm 5.32$ \\
\hline \multirow{5}{*}{ Day $91^{\text {st }}$} & Control & $141.86 \pm 4.19$ & $119.93 \pm 5.66$ & $62.53 \pm 5.49$ & $55.34 \pm 3.94$ \\
\hline & $61.25 \mathrm{mg} / \mathrm{kg}$ & $146.20 \pm 4.94$ & $122.77 \pm 2.43$ & $61.61 \pm 3.07$ & $60.04 \pm 7.80$ \\
\hline & $125 \mathrm{mg} / \mathrm{kg}$ & $144.03 \pm 8.52$ & $116.99 \pm 2.92$ & $56.05 \pm 2.55$ & $64.58 \pm 8.88$ \\
\hline & $250 \mathrm{mg} / \mathrm{kg}$ & $135.34 \pm 3.25$ & $123.68 \pm 5.62$ & $65.77 \pm 4.34$ & $44.83 \pm 4.98$ \\
\hline & $500 \mathrm{mg} / \mathrm{kg}$ & $151.26 \pm 9.46$ & $111.32 \pm 5.77$ & $70.64 \pm 3.53$ & $58.36 \pm 13.72$ \\
\hline \multirow{5}{*}{ Recovery } & Control & $142.45 \pm 1.83$ & $129.01 \pm 5.54$ & $65.44 \pm 1.61$ & $51.21 \pm 2.77$ \\
\hline & $61.25 \mathrm{mg} / \mathrm{kg}$ & $144.81 \pm 3.05$ & $132.83 \pm 2.89$ & $68.16 \pm 1.56$ & $50.09 \pm 3.15$ \\
\hline & $125 \mathrm{mg} / \mathrm{kg}$ & $143.80 \pm 1.62$ & $133.24 \pm 3.96$ & $69.92 \pm 0.62$ & $47.22 \pm 2.23$ \\
\hline & $250 \mathrm{mg} / \mathrm{kg}$ & $141.01 \pm 2.06$ & $123.06 \pm 2.67$ & $70.78 \pm 0.80$ & $45.62 \pm 1.76$ \\
\hline & $500 \mathrm{mg} / \mathrm{kg}$ & $139.41 \pm 2.11$ & $123.09 \pm 3.92$ & $69.66 \pm 1.41$ & $45.12 \pm 2.24$ \\
\hline
\end{tabular}

Values are presented as mean \pm Standard error of mean $(n=5) .{ }^{*} p<0.05$ : Statistically significantly different from control group. 
TABLE VI - Effect of sub-chronic administration of Dryopteris filix-mas ethanol leaf extract on Liver enzymes, alanine aminotransferase (ALT), aspertate aminotransferase (AST), alkaline phosphatase (ALP), total protein and albumin of albino rats

\begin{tabular}{|c|c|c|c|c|c|}
\hline & Treatment & $\operatorname{ALT}(\mathrm{U} / \mathrm{L})$ & $\operatorname{AST}(\mathrm{U} / \mathrm{L})$ & $\operatorname{ALP}(I U / L)$ & $\operatorname{Albumin}(g / d L)$ \\
\hline \multirow{5}{*}{ Baseline } & Control & $16.54 \pm 0.67$ & $38.83 \pm 1.11$ & $46.45 \pm 1.81$ & $3.26 \pm 0.06$ \\
\hline & $61.25 \mathrm{mg} / \mathrm{kg}$ & $17.32 \pm 0.57$ & $35.05 \pm 0.90$ & $48.64 \pm 1.88$ & $3.27 \pm 0.09$ \\
\hline & $125 \mathrm{mg} / \mathrm{kg}$ & $16.26 \pm 0.50$ & $37.45 \pm 1.27$ & $47.41 \pm 1.98$ & $3.26 \pm 0.14$ \\
\hline & $250 \mathrm{mg} / \mathrm{kg}$ & $16.34 \pm 0.55$ & $37.06 \pm 1.50$ & $43.63 \pm 0.35$ & $3.15 \pm 0.07$ \\
\hline & $500 \mathrm{mg} / \mathrm{kg}$ & $15.39 \pm 0.66$ & $36.92 \pm 1.06$ & $48.73 \pm 1.70$ & $3.26 \pm 0.04$ \\
\hline \multirow{5}{*}{ Day $31^{\text {st }}$} & Control & $15.58 \pm 0.86$ & $38.47 \pm 0.94$ & $62.66 \pm 7.71$ & $3.43 \pm 0.23$ \\
\hline & $61.25 \mathrm{mg} / \mathrm{kg}$ & $15.23 \pm 0.85$ & $36.33 \pm 1.98$ & $62.75 \pm 7.40$ & $3.47 \pm 0.27$ \\
\hline & $125 \mathrm{mg} / \mathrm{kg}$ & $12.37 \pm 1.21$ & $32.52 \pm 2.57$ & $51.16 \pm 4.30$ & $3.80 \pm 0.22$ \\
\hline & $250 \mathrm{mg} / \mathrm{kg}$ & $12.82 \pm 1.15$ & $34.76 \pm 1.10$ & $52.63 \pm 6.65$ & $3.92 \pm 0.20$ \\
\hline & $500 \mathrm{mg} / \mathrm{kg}$ & $13.49 \pm 1.59$ & $35.56 \pm 2.10$ & $56.49 \pm 10.32$ & $3.54 \pm 0.26$ \\
\hline \multirow{5}{*}{ Day $61^{\text {st }}$} & Control & $19.78 \pm 0.65$ & $37.72 \pm 0.55$ & $55.22 \pm 0.51$ & $3.69 \pm 0.11$ \\
\hline & $61.25 \mathrm{mg} / \mathrm{kg}$ & $18.93 \pm 0.42$ & $37.94 \pm 0.59$ & $53.66 \pm 6.11$ & $3.72 \pm 0.08$ \\
\hline & $125 \mathrm{mg} / \mathrm{kg}$ & $17.42 \pm 1.85$ & $37.67 \pm 0.56$ & $56.99 \pm 3.35$ & $3.60 \pm 0.14$ \\
\hline & $250 \mathrm{mg} / \mathrm{kg}$ & $16.43 \pm 1.60$ & $37.93 \pm 1.07$ & $56.67 \pm 3.44$ & $3.88 \pm 0.13$ \\
\hline & $500 \mathrm{mg} / \mathrm{kg}$ & $17.12 \pm 1.36$ & $37.62 \pm 0.53$ & $56.08 \pm 0.91$ & $3.67 \pm 0.23$ \\
\hline \multirow{5}{*}{ Day $91^{\text {st }}$} & Control & $17.28 \pm 0.93$ & $35.00 \pm 2.47$ & $45.03 \pm 3.82$ & $3.94 \pm 0.26$ \\
\hline & $61.25 \mathrm{mg} / \mathrm{kg}$ & $16.42 \pm 0.58$ & $39.13 \pm 1.13$ & $40.51 \pm 1.25$ & $4.45 \pm 0.21$ \\
\hline & $125 \mathrm{mg} / \mathrm{kg}$ & $18.24 \pm 1.72$ & $40.60 \pm 2.62$ & $55.10 \pm 1.00 *$ & $4.08 \pm 0.20$ \\
\hline & $250 \mathrm{mg} / \mathrm{kg}$ & $23.36 \pm 1.30^{*}$ & $59.50 \pm 1.92 *$ & $63.70 \pm 2.10^{*}$ & $3.82 \pm 0.13$ \\
\hline & $500 \mathrm{mg} / \mathrm{kg}$ & $30.72 \pm 1.28 *$ & $73.50 \pm 2.21 *$ & $78.02 \pm 3.05^{*}$ & $3.81 \pm 0.28$ \\
\hline \multirow{5}{*}{ Recovery } & Control & $15.92 \pm 1.00$ & $44.49 \pm 1.09$ & $42.30 \pm 2.10$ & $2.66 \pm 0.17$ \\
\hline & $61.25 \mathrm{mg} / \mathrm{kg}$ & $16.16 \pm 1.06$ & $40.66 \pm 1.55$ & $41.34 \pm 3.13$ & $3.57 \pm 0.26$ \\
\hline & $125 \mathrm{mg} / \mathrm{kg}$ & $14.18 \pm 0.94$ & $39.72 \pm 1.68$ & $43.25 \pm 3.68$ & $3.40 \pm 0.23$ \\
\hline & $250 \mathrm{mg} / \mathrm{kg}$ & $14.30 \pm 0.90$ & $40.23 \pm 0.83$ & $37.64 \pm 5.07$ & $2.93 \pm 0.11$ \\
\hline & $500 \mathrm{mg} / \mathrm{kg}$ & $13.26 \pm 1.34$ & $38.80 \pm 1.86$ & $42.23 \pm 3.91$ & $3.51 \pm 0.26$ \\
\hline
\end{tabular}

Values are presented as mean \pm Standard error of mean $(n=5) .{ }^{*} \mathrm{p}<0.05$ : Statistically significantly different from control group.

result which is characterized by predominant glumeruli proliferation with loose Bowmans' space in extensive areas of the kidney in dose dependent manner (Figure 3). Also, this suggests that doses below $250 \mathrm{mg} / \mathrm{kg}$ may be tolerated and safe even beyond 90 days exposure. Significant increase in kidney function parameters and kidney histopathological changes observed on the $91^{\text {st }}$ day were not observed in the recovery studies (Table VIII and Figure 4), suggesting that the injuries attributed to long term exposure of the extract for 90 days was not permanent.

Liver and kidney toxicities observed on $91^{\text {st }}$ day of this study could be attributed to the effects of some secondary metabolites present in the extract of Dryopteris filix-mas as revealed in the phytochemistry result (Table I). Studies have revealed that exposure to high levels of plant secondary metabolites such as tannins, saponins, glycosides and alkaloids could cause hepatorenal toxicity (Netala et al., 2014; Louis et al., 2014; Mariangela et al., 2016). Report had also shown that long term consumption of medicinal plant rich in flavonoids could result to auto-oxidation of reactive oxygen species thereby causing liver and kidney toxicities (Namjoo et al., 2013). Long term intake of cardiac glycosides and diterpenoid glycosides had been reported to cause renal proximal tubule necrosis as well as centrilobular hepatic necrosis 

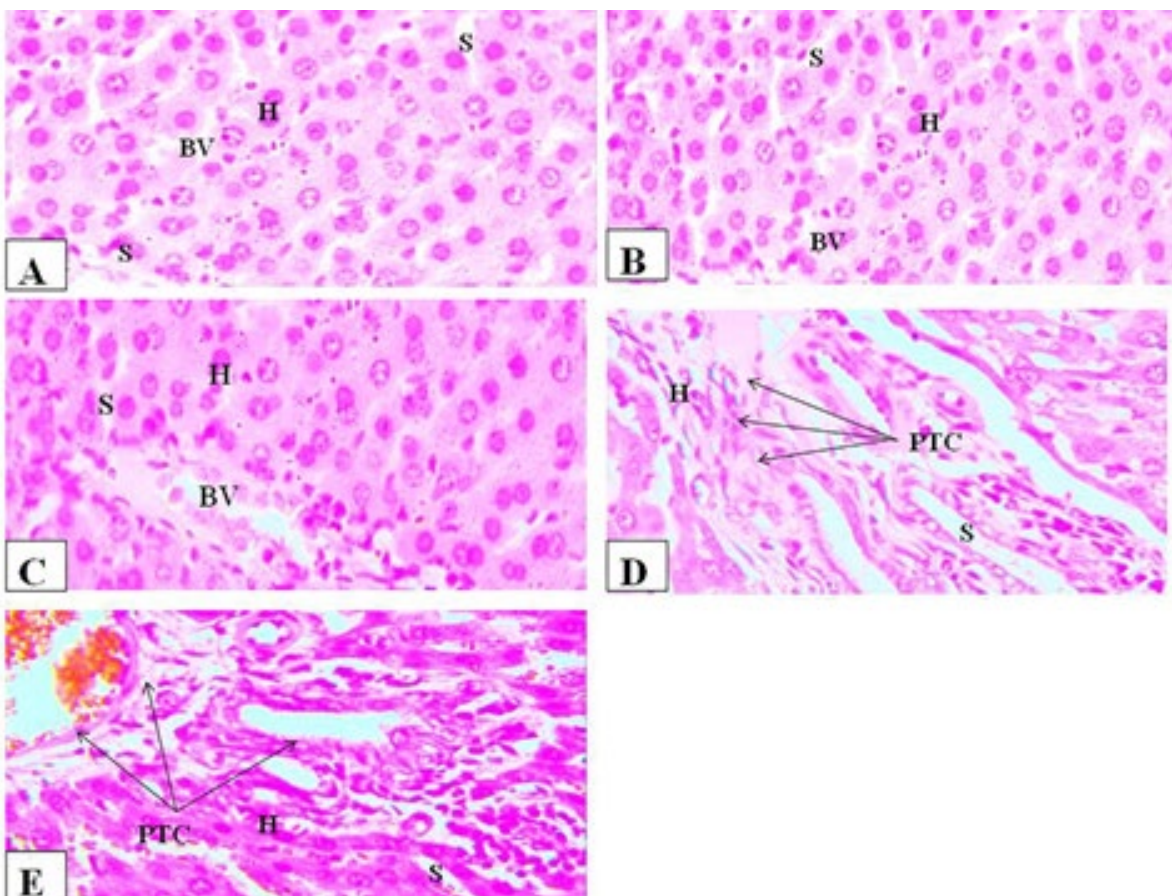

FIGURE 1 - $91^{\text {st }}$ day liver sections photomicrographs showing; Plate A (Control), Plate B (62.5 mg/kg), Plate C (125 mg/kg), Plate D (250 mg/kg), Plate E (500 mg/kg). H: Hepatocytes, BV: Blood vessel, S: Sinosoid. PTC: Portal triad congestion).
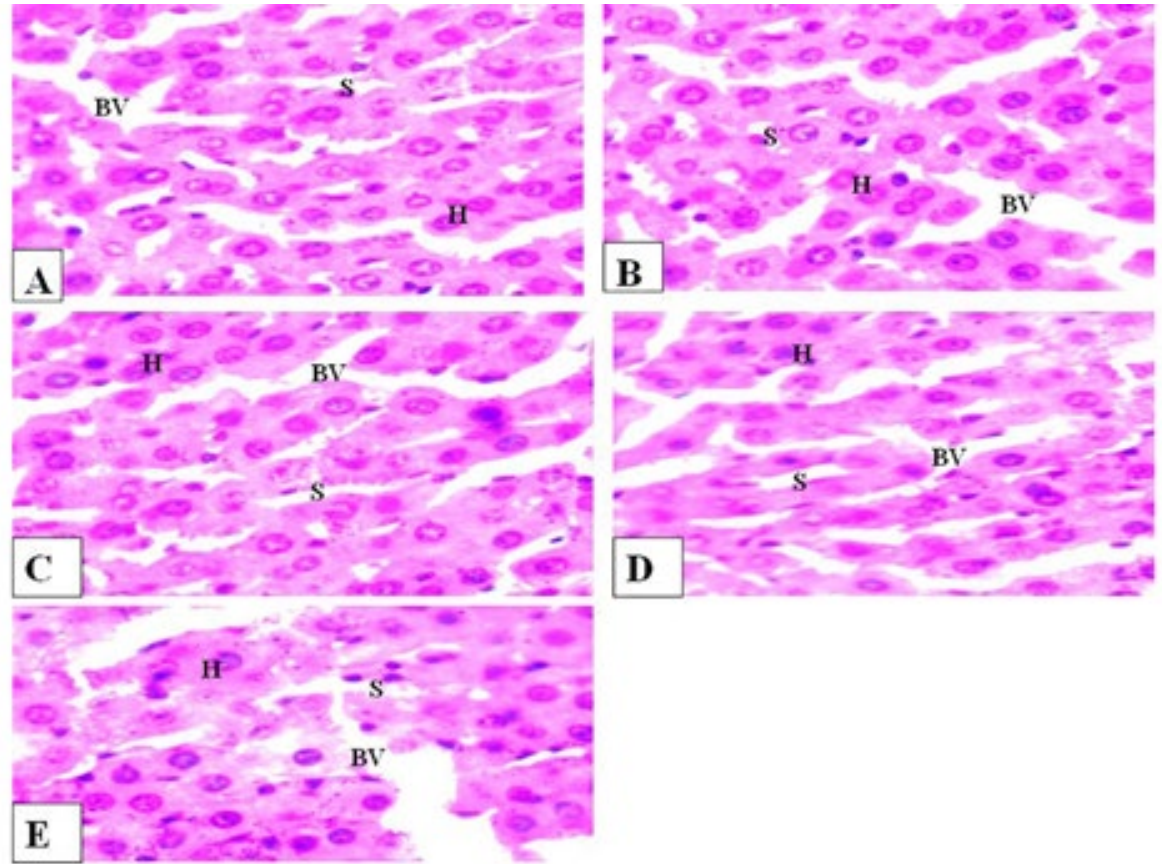

FIGURE 2 - Recovery liver sections photomicrographs showing; Plate A (Control), Plate B (62.5 mg/kg), Plate C (125 mg/kg), Plate D $(250 \mathrm{mg} / \mathrm{kg})$, Plate E (500 mg/kg). H: Hepatocytes disposed in sheet. S: sinusoids, BV: Blood vessel.

in animals (Chikezie, Ibegbulem, Mbagwu, 2015). Nearly all alkaloids from plants are believed to be responsible for nitrogen secretion (like urea and uric acid in animals) and could be toxic when ingested in large quantity (Olivoto et al., 2017). Builders, Isichie, Aguiyi (2012) also revealed that presence of tannins in stem bark extract of Parkia biglobosa may be responsible for its liver and kidney damage. Osano et al. (2016) also revealed that saponins and tannins in methanol leaf extracts of Prosopis juliflora may be associated with its hepetorenal toxicity. From this study, presence of tannins, saponins, glycosides, flavonoids and alkaloids in Dryopteris filix-mas leaf 
TABLE VII - Effect of sub-chronic administration of Dryopteris filix-mas ethanol leaf extract on sodium, potassium, chloride and total protein of albino rats

\begin{tabular}{|c|c|c|c|c|c|}
\hline & Treatment & $\begin{array}{l}\text { Sodium } \\
(\mathrm{mEq} / \mathrm{L})\end{array}$ & Potassium $(\mathrm{mEq} / \mathrm{L})$ & $\begin{array}{l}\text { Chloride } \\
(\mathrm{mEq} / \mathrm{L})\end{array}$ & $\begin{array}{l}\text { Total protein } \\
\text { (g/dL) }\end{array}$ \\
\hline \multirow{5}{*}{ Baseline } & Control & $144.77 \pm 2.68$ & $4.47 \pm 0.13$ & $108.47 \pm 2.13$ & $4.23 \pm 0.09$ \\
\hline & $61.25 \mathrm{mg} / \mathrm{kg}$ & $146.37 \pm 5.63$ & $4.28 \pm 0.21$ & $104.24 \pm 1.53$ & $4.61 \pm 0.19$ \\
\hline & $125 \mathrm{mg} / \mathrm{kg}$ & $144.00 \pm 2.75$ & $4.49 \pm 0.21$ & $105.11 \pm 3.04$ & $4.64 \pm 0.59$ \\
\hline & $250 \mathrm{mg} / \mathrm{kg}$ & $145.27 \pm 6.78$ & $4.55 \pm 0.09$ & $106.24 \pm 2.72$ & $4.24 \pm 0.04$ \\
\hline & $500 \mathrm{mg} / \mathrm{kg}$ & $146.99 \pm 3.46$ & $4.39 \pm 0.19$ & $101.58 \pm 3.65$ & $4.27 \pm 0.24$ \\
\hline \multirow{5}{*}{ Day $31^{\mathrm{st}}$} & Control & $143.73 \pm 0.69$ & $4.08 \pm 0.29$ & $96.52 \pm 2.75$ & $4.94 \pm 0.30$ \\
\hline & $61.25 \mathrm{mg} / \mathrm{kg}$ & $145.28 \pm 1.66$ & $3.95 \pm 0.23$ & $97.15 \pm 3.70$ & $4.93 \pm 0.30$ \\
\hline & $125 \mathrm{mg} / \mathrm{kg}$ & $142.17 \pm 1.81$ & $4.34 \pm 0.22$ & $92.22 \pm 1.55$ & $4.80 \pm 0.15$ \\
\hline & $250 \mathrm{mg} / \mathrm{kg}$ & $142.42 \pm 2.36$ & $4.18 \pm 0.23$ & $86.88 \pm 1.66$ & $5.01 \pm 0.16$ \\
\hline & $500 \mathrm{mg} / \mathrm{kg}$ & $139.96 \pm 2.45$ & $4.43 \pm 0.20$ & $90.95 \pm 0.94$ & $4.87 \pm 0.08$ \\
\hline \multirow{5}{*}{ Day $61^{\text {st }}$} & Control & $131.17 \pm 1.66$ & $5.21 \pm 0.14$ & $95.99 \pm 3.39$ & $4.54 \pm 0.26$ \\
\hline & $61.25 \mathrm{mg} / \mathrm{kg}$ & $130.96 \pm 1.79$ & $4.92 \pm 0.31$ & $93.60 \pm 3.68$ & $4.56 \pm 0.20$ \\
\hline & $125 \mathrm{mg} / \mathrm{kg}$ & $133.42 \pm 4.83$ & $4.33 \pm 0.76$ & $88.72 \pm 4.10$ & $4.24 \pm 0.19$ \\
\hline & $250 \mathrm{mg} / \mathrm{kg}$ & $141.63 \pm 3.12$ & $3.81 \pm 0.04$ & $96.23 \pm 2.82$ & $4.73 \pm 0.41$ \\
\hline & $500 \mathrm{mg} / \mathrm{kg}$ & $141.26 \pm 3.75$ & $3.93 \pm 0.53$ & $100.20 \pm 3.40$ & $4.42 \pm 0.29$ \\
\hline \multirow{5}{*}{ Day $91^{\text {st }}$} & Control & $132.01 \pm 1.69$ & $3.64 \pm 0.22$ & $93.26 \pm 3.23$ & $4.78 \pm 0.34$ \\
\hline & $61.25 \mathrm{mg} / \mathrm{kg}$ & $134.13 \pm 1.28$ & $3.68 \pm 0.14$ & $94.72 \pm 1.31$ & $4.96 \pm 0.08$ \\
\hline & $125 \mathrm{mg} / \mathrm{kg}$ & $129.49 \pm 0.35$ & $4.35 \pm 0.09$ & $84.15 \pm 3.52$ & $5.01 \pm 0.25$ \\
\hline & $250 \mathrm{mg} / \mathrm{kg}$ & $139.12 \pm 6.68$ & $3.14 \pm 0.38$ & $85.94 \pm 4.76$ & $5.13 \pm 0.26$ \\
\hline & $500 \mathrm{mg} / \mathrm{kg}$ & $136.52 \pm 1.12$ & $4.03 \pm 0.50$ & $92.39 \pm 2.68$ & $5.26 \pm 0.10$ \\
\hline \multirow{5}{*}{ Recovery } & Control & $134.23 \pm 3.83$ & $3.96 \pm 0.26$ & $98.03 \pm 9.67$ & $4.06 \pm 0.04$ \\
\hline & $61.25 \mathrm{mg} / \mathrm{kg}$ & $138.26 \pm 5.11$ & $3.42 \pm 0.07$ & $106.81 \pm 4.88$ & $4.11 \pm 0.06$ \\
\hline & $125 \mathrm{mg} / \mathrm{kg}$ & $136.02 \pm 3.80$ & $4.28 \pm 0.48$ & $102.38 \pm 3.85$ & $4.08 \pm 0.05$ \\
\hline & $250 \mathrm{mg} / \mathrm{kg}$ & $143.98 \pm 1.75$ & $5.15 \pm 0.22$ & $85.12 \pm 6.37$ & $4.01 \pm 0.08$ \\
\hline & $500 \mathrm{mg} / \mathrm{kg}$ & $141.74 \pm 3.31$ & $3.93 \pm 0.53$ & $91.81 \pm 3.88$ & $4.10 \pm 0.09$ \\
\hline
\end{tabular}

Values are presented as mean \pm Standard error of mean $(n=5) . p>0.05$ : Not statistically significantly different from control group.

extract may be responsible for the liver and kidney toxicities observed on $91^{\text {st }}$ day.

Non-significant difference $(\mathrm{p}>0.05)$ in body weight gain of animals treated with various doses of the extract for 90 days (Table IX) suggests that subchronic administration of the extract does not contribute to body weight reduction. Ekpenyong, Akpan, Udoh (2012) reported that high level of hydrolysable tannins (non-digestable form) from phytochemicals could cause appetite suppression and body weight reduction when compared to condensed tannins.
Non-significant changes in organs weights on $91^{\text {st }}$ day and in recovery studies (Tables X) indicate that the extract may not be associated with hypertrophy or hyperplasia. Studies by Otunola, Afolayan (2017) revealed that increase or decrease in organ weights may be associated with marked organ toxicity.

Studies by Moke, Ilodigwe, Erhirhie (2015) revealed that evaluation of ulcerogenic properties of medicinal plants could aid in validating their safety profile relating to long term use. Absence of ulcerations in the stomach mucosa of rats that were exposed to the extract for 90 days 
TABLE VIII - Effect of sub-chronic administration of Dryopteris filix-mas ethanol leaf extract on urea, creatinine and calcium of albino rats

\begin{tabular}{|c|c|c|c|c|}
\hline & Treatment & Urea (mg/dL) & Creatinine (mg/dL) & Calcium (mg/dL) \\
\hline \multirow{5}{*}{ Baseline } & Control & $19.88 \pm 0.86$ & $4.36 \pm 0.21$ & $10.80 \pm 0.62$ \\
\hline & $61.25 \mathrm{mg} / \mathrm{kg}$ & $20.82 \pm 0.45$ & $4.28 \pm 0.16$ & $8.93 \pm 0.45$ \\
\hline & $125 \mathrm{mg} / \mathrm{kg}$ & $19.86 \pm 0.62$ & $3.95 \pm 0.10$ & $9.95 \pm 0.46$ \\
\hline & $250 \mathrm{mg} / \mathrm{kg}$ & $21.15 \pm 0.49$ & $4.00 \pm 0.17$ & $10.51 \pm 0.31$ \\
\hline & $500 \mathrm{mg} / \mathrm{kg}$ & $20.40 \pm 0.59$ & $4.19 \pm 0.21$ & $9.73 \pm 0.48$ \\
\hline \multirow{5}{*}{ Day $31^{\text {st }}$} & Control & $19.65 \pm 0.45$ & $5.64 \pm 0.19$ & $9.95 \pm 0.27$ \\
\hline & $61.25 \mathrm{mg} / \mathrm{kg}$ & $20.17 \pm 0.61$ & $5.29 \pm 0.33$ & $9.50 \pm 0.23$ \\
\hline & $125 \mathrm{mg} / \mathrm{kg}$ & $22.08 \pm 0.82$ & $5.46 \pm 0.22$ & $9.24 \pm 0.12$ \\
\hline & $250 \mathrm{mg} / \mathrm{kg}$ & $19.57 \pm 0.71$ & $5.67 \pm 0.10$ & $9.48 \pm 0.37$ \\
\hline & $500 \mathrm{mg} / \mathrm{kg}$ & $20.29 \pm 1.11$ & $5.75 \pm 0.24$ & $9.94 \pm 0.33$ \\
\hline \multirow{5}{*}{ Day $61^{\text {st }}$} & Control & $19.06 \pm 1.61$ & $4.70 \pm 0.32$ & $11.05 \pm 0.77$ \\
\hline & $61.25 \mathrm{mg} / \mathrm{kg}$ & $19.82 \pm 1.75$ & $4.32 \pm 0.28$ & $10.74 \pm 0.66$ \\
\hline & $125 \mathrm{mg} / \mathrm{kg}$ & $20.35 \pm 0.78$ & $4.09 \pm 0.14$ & $10.63 \pm 0.44$ \\
\hline & $250 \mathrm{mg} / \mathrm{kg}$ & $19.65 \pm 1.01$ & $4.00 \pm 0.24$ & $10.93 \pm 0.36$ \\
\hline & $500 \mathrm{mg} / \mathrm{kg}$ & $21.12 \pm 1.65$ & $4.60 \pm 0.22$ & $10.88 \pm 0.30$ \\
\hline \multirow{5}{*}{ Day $91^{\text {st }}$} & Control & $19.83 \pm 0.18$ & $3.70 \pm 0.08$ & $9.34 \pm 0.71$ \\
\hline & $61.25 \mathrm{mg} / \mathrm{kg}$ & $19.96 \pm 0.40$ & $3.60 \pm 0.06$ & $9.35 \pm 0.48$ \\
\hline & $125 \mathrm{mg} / \mathrm{kg}$ & $20.80 \pm 0.40$ & $4.00 \pm 0.06$ & $9.51 \pm 0.66$ \\
\hline & $250 \mathrm{mg} / \mathrm{kg}$ & $23.79 \pm 0.29 *$ & $4.77 \pm 0.04 *$ & $9.32 \pm 0.19$ \\
\hline & $500 \mathrm{mg} / \mathrm{kg}$ & $24.97 \pm 0.18^{*}$ & $5.54 \pm 0.13^{*}$ & $10.40 \pm 0.19$ \\
\hline \multirow{5}{*}{ Recovery } & Control & $18.36 \pm 0.55$ & $4.38 \pm 0.54$ & $8.05 \pm 0.34$ \\
\hline & $61.25 \mathrm{mg} / \mathrm{kg}$ & $18.78 \pm 0.38$ & $5.15 \pm 0.60$ & $8.49 \pm 0.74$ \\
\hline & $125 \mathrm{mg} / \mathrm{kg}$ & $18.32 \pm 0.15$ & $4.90 \pm 0.42$ & $7.74 \pm 0.32$ \\
\hline & $250 \mathrm{mg} / \mathrm{kg}$ & $19.20 \pm 0.20$ & $5.50 \pm 0.45$ & $8.50 \pm 0.51$ \\
\hline & $500 \mathrm{mg} / \mathrm{kg}$ & $18.61 \pm 0.22$ & $4.70 \pm 0.31$ & $9.14 \pm 0.37$ \\
\hline
\end{tabular}

Values are presented as mean \pm Standard error of mean $(n=5) .{ }^{*} p<0.05$ : Statistically significantly different from control group.

(Table XI) suggest that the extract is not be ulcerogenic in the stomach mucosa on long term use.

\section{CONCLUSIONS}

Acute toxicity study revealed that ethanol leaf extract of Dryopteris filix-mas may be safe on single dose exposure. However, 90 days repeated exposure of animals to 250 and $500 \mathrm{mg} / \mathrm{kg}$ doses of this extract may cause reduction in leukocyte counts and hepatorenal injuries, which may be reversible.
Thus, caution should be exercised by consumers who use high doses of the extract over a long period of time.

\section{CONFLICTS OF INTEREST}

The authors declare no conflict of interest.

\section{ACKNOWLEDGMENTS}

Nil. 


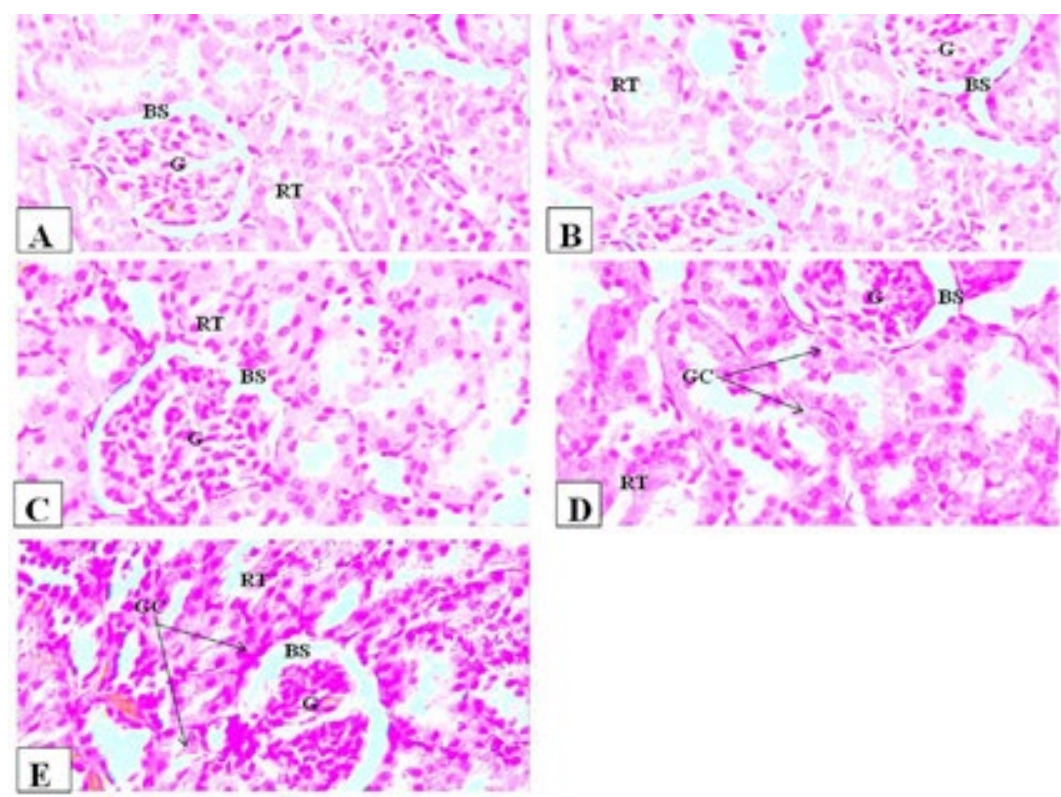

FIGURE 3 - 91 $1^{\text {st }}$ day kidney sections photomicrographs showing; Plate A (Control), Plate B (62.5 mg/kg), Plate C (125 mg/kg), Plate D $(250 \mathrm{mg} / \mathrm{kg})$, Plate E (500 mg/kg). G: Glomeruli, BS: Bowman's space, RT: Renal tubule. GC: Glomeruli congestion.

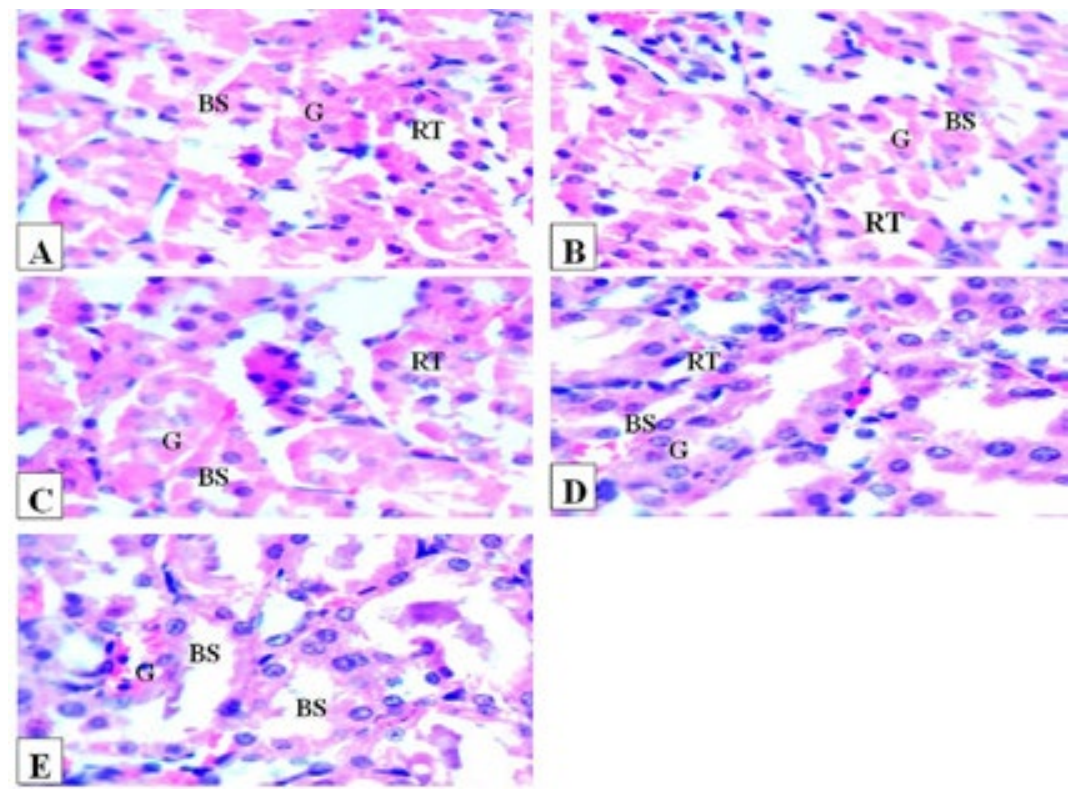

FIGURE 4 - Recovery kidney sections photomicrographs showing; Plate A(Control), Plate B (62.5 mg/kg), Plate C (125 mg/kg), Plate D $(250 \mathrm{mg} / \mathrm{kg})$, Plate E (500 mg/kg). G: Glomeruli, BS: Bowman's space, RT: Renal tubule.

TABLE IX - Effect of sub-chronic administration of Dryopteris filix-mas ethanol leaf extract on body weight of albino rats.

\begin{tabular}{lcccc}
\hline & \multicolumn{4}{c}{ Body weight gain (\%) } \\
\cline { 2 - 5 } & $\mathbf{3 1}^{\text {st }} \mathbf{d a y}$ & $\mathbf{6 1}^{\text {st }}$ day & $\mathbf{9 1}^{\text {st }}$ day & Recovery \\
\hline Control & $35.02 \pm 2.00$ & $37.97 \pm 5.11$ & $57.22 \pm 2.99$ & $48.34 \pm 9.89$ \\
$61.25 \mathrm{mg} / \mathrm{kg}$ & $33.87 \pm 1.72$ & $38.28 \pm 4.95$ & $46.25 \pm 6.41$ & $63.57 \pm 3.93$ \\
$125 \mathrm{mg} / \mathrm{kg}$ & $36.51 \pm 2.20$ & $51.95 \pm 3.57$ & $60.73 \pm 3.48$ & $52.44 \pm 10.51$ \\
$250 \mathrm{mg} / \mathrm{kg}$ & $38.37 \pm 3.59$ & $42.32 \pm 6.48$ & $55.69 \pm 5.35$ & $51.81 \pm 9.29$ \\
$500 \mathrm{mg} / \mathrm{kg}$ & $35.79 \pm 2.42$ & $47.71 \pm 6.07$ & $57.87 \pm 5.84$ & $60.53 \pm 5.32$ \\
\hline
\end{tabular}

Values are presented as mean \pm Standard error of mean $(n=5) . p>0.05$ : Not statistically significantly different from control group. 
TABLE X - Effect of sub-chronic administration of Dryopteris filix-mas ethanol leaf extract on relative organs weight of albino rats

\begin{tabular}{ccccccc}
\hline & & Liver (\%) & Kidney (\%) & Heart (\%) & Spleen (\%) & Lung (\%) \\
\hline \multirow{4}{*}{ Day 91 ${ }^{\text {st }}$} & Control & $2.96 \pm 0.06$ & $0.71 \pm 0.02$ & $0.44 \pm 0.03$ & $0.39 \pm 0.02$ & $0.67 \pm 0.02$ \\
& $61.25 \mathrm{mg} / \mathrm{kg}$ & $3.14 \pm 0.11$ & $0.75 \pm 0.01$ & $0.47 \pm 0.01$ & $0.39 \pm 0.02$ & $0.70 \pm 0.03$ \\
& $125 \mathrm{mg} / \mathrm{kg}$ & $3.10 \pm 0.10$ & $0.73 \pm 0.01$ & $0.46 \pm 0.01$ & $0.40 \pm 0.02$ & $0.71 \pm 0.03$ \\
& $250 \mathrm{mg} / \mathrm{kg}$ & $3.10 \pm 0.03$ & $0.70 \pm 0.02$ & $0.41 \pm 0.03$ & $0.34 \pm 0.01$ & $0.73 \pm 0.04$ \\
& $500 \mathrm{mg} / \mathrm{kg}$ & $3.09 \pm 0.03$ & $0.71 \pm 0.03$ & $0.45 \pm 0.05$ & $0.42 \pm 0.01$ & $0.71 \pm 0.02$ \\
\hline \multirow{3}{*}{ Recovery } & Control & $3.14 \pm 0.12$ & $0.65 \pm 0.04$ & $0.39 \pm 0.03$ & $0.31 \pm 0.01$ & $0.87 \pm 0.11$ \\
& $61.25 \mathrm{mg} / \mathrm{kg}$ & $2.93 \pm 0.25$ & $0.59 \pm 0.02$ & $0.33 \pm 0.03$ & $0.30 \pm 0.01$ & $0.75 \pm 0.12$ \\
& $125 \mathrm{mg} / \mathrm{kg}$ & $2.77 \pm 0.10$ & $0.68 \pm 0.03$ & $0.32 \pm 0.01$ & $0.35 \pm 0.01$ & $0.68 \pm 0.03$ \\
& $250 \mathrm{mg} / \mathrm{kg}$ & $2.77 \pm 0.10$ & $0.62 \pm 0.02$ & $0.34 \pm 0.03$ & $0.29 \pm 0.02$ & $0.86 \pm 0.20$ \\
& $500 \mathrm{mg} / \mathrm{kg}$ & $2.78 \pm 0.10$ & $0.62 \pm 0.02$ & $0.29 \pm 0.01$ & $0.36 \pm 0.02$ & $0.78 \pm 0.10$ \\
\hline
\end{tabular}

Values are presented as mean \pm Standard error of mean $(n=5) . p>0.05$ : Statistically significantly different from control group

TABLE XI - Effects of sub-chronic administration of Dryopteris filix-mas leaf extract on stomach mucosa of albino rats

\begin{tabular}{lcc}
\hline \multirow{2}{*}{ Dose $(\mathbf{m g} / \mathbf{k g})$} & \multicolumn{2}{c}{ Ulcer score } \\
\cline { 2 - 3 } & Day 91 $^{\text {st }}$ & Recovery \\
\hline Control & $0.00 \pm 0.00$ & $0.00 \pm 0.00$ \\
62.5 & $0.00 \pm 0.00$ & $0.00 \pm 0.00$ \\
125 & $0.00 \pm 0.00$ & $0.00 \pm 0.00$ \\
250 & $0.00 \pm 0.00$ & $0.00 \pm 0.00$ \\
500 & $0.00 \pm 0.00$ & $0.00 \pm 0.00$ \\
\hline
\end{tabular}

Values are presented as mean \pm Standard error of mean (SEM), $\mathrm{n}=5$.

\section{REFERENCE}

Aksel B. Bioactive compounds in plants - benefits and risks for man and animals. [Proceedings from a symposium held at The Norwegian Academy of Science and Letters, Oslo, 13 - 14 November 2008 Novus forlag, Oslo 2010] [Cited 2017 Dec 10]. http://www.dnva.no/geomed); http://www.dnva.no/binfil/ download.php?tid=48677.

Azubike NC, Okwuosa CN, Achukwu PU, Maduka TC, Chike O. Acute toxicity and histopathological effects of crude aqueous extract of Jatropha curcas leaves in mice. J Med Plants Res. 2015;9(7):340-6.

Bancroft JD, Gamble M. Theory and practice of histological techniques. Edinburgh: Churchill Livingstone; 2002. p.16-64.
Bashir L, Oluwatosin KS, Agboola AR, Ibrahim AO, Adeniyi AK, Prince CO. Effect of methanol extract of Telfairia occcidentalis on haematological parameters in Wister rats. J Med Sci. 2015;15(5):246-50.

Builders MI, Isichie CO, Aguiyi JC. Toxicity studies of the extracts of Parkia biglobosa Stem Bark in rats. Br J Pharm Res. 2012;2(1):1-16.

Chikezie PC, Ibegbulem CO, Mbagwu FN. Medicinal potentials and toxicity concerns of bioactive principles. Med Aromat Plants. 2015;4(3):1-15.

Debelo N, Afework M, Debella A, Makonnen E, Ergete W. Assessment of hematological, biochemical and histopathological effects of acute and sub-chronic administration of the aqueous leaves extract of Thymus schimperi in rats. J Clin Toxicol. 2016;6(2):1-9.

Ekpenyong CE, Akpan EE, Udoh NS. Phytochemistry and toxicity studies of Telfairia Occidentalis aqueous leaves extract on liver biochemical indices in Wistar rats. AJMS Am J Med Med Sci. 2012; 2(5): 103-10.

Harborne JB. Phytochemicals methods. London: Chapman and Hall; 1973. p.49-188.

Ilodigwe EE, Akah PA, Nworu, CS. Evaluation of the acute and subchronic toxicities of ethanol leaf extract of Spathodea campanulata P. Beauv. Int J Appl Res. 2010;3(2):17-21. 
Kayode AA, Chinedu I, Chukwuma SE, Zuhairah IM. Effects of ethanolic extracts of Datura metel on blood lipid profile of male albino rats. Int J Sci Rep. 2016;2(10):248-52.

Kumar G, Karthik L, Rao VB. Haemolytic activity of Indian medicinal plants towards erythrocytes: an in vitro study. Elixir Appl Bot. 2011;40:5534-7.

Louis AA, Christian AEK, Patrick GA, Kwesi BM. Toxicity assessment of Erythrophleum ivorense and Parquetina nigrescens. Toxicol Rep. 2014;1:411-20.

Mariangela M, Filomena C, Fabrizio A, Giancarlo AS. Effects of Saponins on lipid metabolism: a review of potential health benefits in the treatment of obesity. Molecules. 2016;21(10 E1419):1-20.

Moke EG, Ilodigwe EE, Erhirhie OE. Evaluation of the ulcerogenic potential of the aqueous extract of Spondias mombin and Custus afer. IJAPBC. 2015;4(2):282-6.

Muhammad K, Mohd SM, Pinaki S, Moklesur RS, Arindam D, Sreemoy KD. Evaluation of the acute and sub-acute toxicity of the ethanolic extract of Pericampylus glaucus (Lam.) Merr. in BALB/c mice. J Acute Dis. 2015; 4(4):309-15.

Namjoo AR, MirVakili M, Shirzad H, Faghani M. Biochemical, liver and renal toxicities of Melissa officinals hydroalcoholic extract on balb/C mice. J HerbMed Pharmacol. 2013;2(2):35-40.

Netala VR, Ghosh SB, Bobbu P, Anitha D, Tartte V. Triterpenoid saponins: a review on biosynthesis, applications and mechanism of their action. Int J Pharm Pharm Sci. 2014;7(1):24-8.

Obi H, Ilodigwe EE, Ajaghaku DL, Okonta JM. An evaluation of acute and subchronic toxicities of a Nigerian polyherbal antidiabetic remedy. Int J Pharm Sci Res. 2012;3(9):3131-5.

Ogbonnaya EA, Uadia PO. Effect of sub acute exposure to Telfairia occidentalis root, pod and stem extracts on some liver and renal function parameters in rats. $\mathrm{Br} \mathrm{J}$ Pharm Res. 2016;11(3):1-8

Ogbonnia SO, Mbaka GO, Anyika EN, Emordi JE, Nwakakwa N. An evaluation of acute and subchronic toxicities of a Nigerian polyherbal tea remedy. Pak J Nutr. 2011;10(11):1022-8.

Olivoto T, Maicon N, Ivan RC, Diego NF, Vinícius JS, Mauricio $\mathrm{F}$, et al. Plant secondary metabolites and its dynamical systems of induction in response to environmental factors: a review. Afr J Agric Res. 2017;12(2):71-84.
Osano KO, Nyamai DW, Ogola PE, Ouko RO, Arika WM. Evaluation of in vivo toxicity of dichloromethane: methanolic leaf extracts of prosopis juliflora in female Wistar albino rats. J Drug Metab Toxicol. 2016;7(1):1-11.

Otunola GA, Afolayan AJ. Assessment of oral safety profile of aqueous extract blend of three medicinal spices in Wistar rats. Trop J Pharm Res. 2017;16(1):91-9.

Randhawa MA. Calculation of $\mathrm{LD}_{50}$ value from the method of Miller and Tainter. J Ayub Med Coll Abbottabad. 2009;21(3):13.

Sekendar AM, Mostafa K, Raihan MO, Rahman MK, Hossain MA, Alam MS. Antioxidant and cytotoxic activities of methanolic extract of Dryopteris filix-mas (L.) Schott leaves. Int J Drug Dev Res. 2012;4(2):223-9.

Soare LC, Ferdes M, Stefanov S, Denkova Z, Nicolova R, Denev $P$, et al. Antioxidant activity, polyphenols content and antimicrobial activity of several native pteridophytes of Romania. Not Bot Horti Agrobo. 2012;40(1):53-7.

Sofowora A. Medicinal plants and traditional medicine in Africa. Ibadan: Spectrum Books; 1993. p.191-289.

Trease GE, Evans WC. Pharmacognosy. $11^{\text {th }}$.ed. London: Bailliere Tindall; 1989. p.45-50.

Unakalamba BC, Ozougwu JC, Ejere VC. Preliminary evaluation of the haematological effects of Picralima nitida saponin extracts on Rattus novergicus. IJBBS. 2013;2(2):28-32.

Urban J, Tauchen J, Langrova I, Kokoska L. In vitro motility inhibition effect of Czech medicinal plant extracts on Chabertia ovina adults. J Anim Plant Sci. 2014;21(2);3293-302.

Uwumarongie HO, Enike MA, Bafor EE. Pharmacognostic evaluation and gastrointestinal activity of Dryopteris filixmas (L.) schott (Dryopteridaceae). Ewemen J Herbal Chem Pharmacol Res. 2016;2(1):19-25.

Yadav RNS, Agarwala M. Phytochemical analysis of some medicinal plants. J Phytol. 2011;3(12):10-4.

Received for publication on $17^{\text {th }}$ February 2018 Accepted for publication on $28^{\text {th }}$ May 2018 